\title{
Asplenia congénita y purpura fulminans neumocóccico en paciente pediátrico. Reporte de caso con necropsia y revisión del tema
}

\author{
Katalina Bertrán S., Alejandro Donoso F., Pablo Cruces R., Franco Díaz R. y Daniela Arriagada S.
}

\section{Congenital asplenia and pneumococcal purpura fulminans in a pediatric patient. Case report with pathological findings and review}

Absent or defective splenic function is associated with a high risk of fulminant bacterial infections, especially due to encapsulated bacteria. Not knowing this condition may delay medical treatment. Streptococcus pneumoniae is the leading cause of sepsis in these patients. Asplenic patients are at high risk for septic shock and eventually purpura fulminans, a life-threatening condition. We report the case of a 3 years old girl, with mitral stenosis and recurrent pneumonia that was admitted due to fever but in the next few hours presented hypotension, purpura and livedo reticularis. Laboratory test showed leucopenia $\left(3.400 / \mathrm{mm}^{3}\right)$, bandemia (43\% of immature forms), thrombocytopenia, hypoprothombinemia and severe lactic acidosis ( $\mathrm{ph}$ : 7.0 and lactic acid $11 \mathrm{mmol} / \mathrm{l})$. The patient developed septic shock and multiorganic failure. Mechanical ventilation, volume resuscitation, vasoactive drugs and antibiotic therapy was initiated. Ultrasound was performed on the second day, demostrating asplenia. Peripheral blood smear showed Howell-Jolly bodies. Patient had a positive blood culture for penicillin-resistant Streptococcus pneumoniae (serotype 19F). Patient died of intracerebral hemorrhage after 8 days of admission. Necropsy confirmed asplenia and bilateral suprarenal haemorrhage. Absence of spleen can lead to life threatening infections, it is important to recognize it because vaccination and antibiotic prophylaxis can provide life-saving protection. This case provides a reminder to pursue asplenia as a potential underlying mechanism for invasive bacterial infection in children.

Key words: Asplenia, Streptococcus pneumoniae, pneumococcal sepsis, purpura fulminans, HowellJolly bodies.

Palabras clave: Asplenia, Streptococcus pneumoniae, sepsis neumocóccica, purpura fulminans, corpúsculos de Howell-Jolly.

\section{Introducción}

$\mathrm{L}$ a asplenia congénita es infrecuente, su incidencia exacta se desconoce, ya que es comúnmente sub-diagnosticada ${ }^{1-3}$. Estudios en neonatos con malformación cardíaca describen la asociación con asplenia hasta en $3 \%{ }^{4}$. El diagnóstico de asplenia congénita es difícil y requiere un alto índice de sospecha ya que su reconocimiento precoz puede cambiar la morbi-mortalidad asociada esta condición.

La presencia de una función esplénica reducida se ve reflejada en una disminución del aclaramiento de antígenos, tanto extracelulares como intracelulares, en una disminución de la respuesta a nuevos antígenos (lipopolisacáridos), en la alteración de la fagocitosis bacteriana y una disminución de los niveles de properdina $y$ tuftsina ${ }^{5}$.
Purpura fulminans (PF) es una patología poco frecuente, de extrema gravedad y alta mortalidad. Se caracteriza fundamentalmente por la repentina aparición y rápida progresión de extensas equimosis cutáneas purpúricas, eventos trombóticos y consumo acelerado de factores de coagulación, produciéndose coagulación intravascular diseminada en el contexto de una sepsis. La etiología más común, en la edad pediátrica es Neisseria meningitidis; sin embargo, en pacientes asplénicos éstas son mayormente causadas por Streptococcus pneumoniae . $^{6}$

El propósito de este artículo es comunicar un caso clínico inhabitual, sin reportes nacionales previos, de una paciente con asplenia congénita que desarrolló PF neumocóccico de resultado fatal, que podría haber sido prevenido en forma efectiva de haberse tenido una conducta pro-activa en su búsqueda. Se efectúa una revisión del tema.
Facultad de Medicina Clínica Alemana-Universidad del Desarrollo, Santiago, Chile Escuela de Medicina (KBS) Departamento de Pediatría (DAS) Hospital Padre Hurtado, Santiago, Chile

Área de Cuidados Críticos (ADF, PCR, FDR)

Recibido: 14 abril 2008 Aceptado: 10 noviembre 2008

Correspondencia a: Alejandro Donoso Fuentes adonoso@hurtadohosp.cl 
Figura 1. Se observa palma de la mano derecha con lesiones equimóticas y violáceas en forma estrellada, características de purpura fulminans.

\section{Caso Clínico}

Paciente de sexo femenino, con 3 años 10 meses de edad y antecedente de estenosis mitral grave y neumonías a repetición, que motivaron nueve hospitalizaciones en tres años, ninguna de ellas de carácter grave o que ameritase terapia en UCI. Ingresó al área de cuidados básicos con diagnóstico de síndrome febril en estudio, con historia de decaimiento y fiebre de corta duración. Desarrolló a las pocas horas shock y lesiones purpúricas en la zona inguinal. Fue trasladada al área de cuidados críticos (ACC) con compromiso de conciencia, febril $\left(\mathrm{T}^{\circ} 38,2^{\circ} \mathrm{C}\right)$, hipotensión arterial (presión arterial media $56 \mathrm{mmHg}$ ), púrpura y livedo reticularis generalizado (Figura 1). En sus exámenes de ingreso a ACC, destacaba una lactacidemia grave (gases venosos con $\mathrm{pH} 7,0, \mathrm{BE}-18$, lactato $11 \mathrm{mmol} / \mathrm{l}$, (VN = 0,5-2,2 mmol/l), en el hemograma había leucopenia con desviación izquierda (recuento leucocitario en
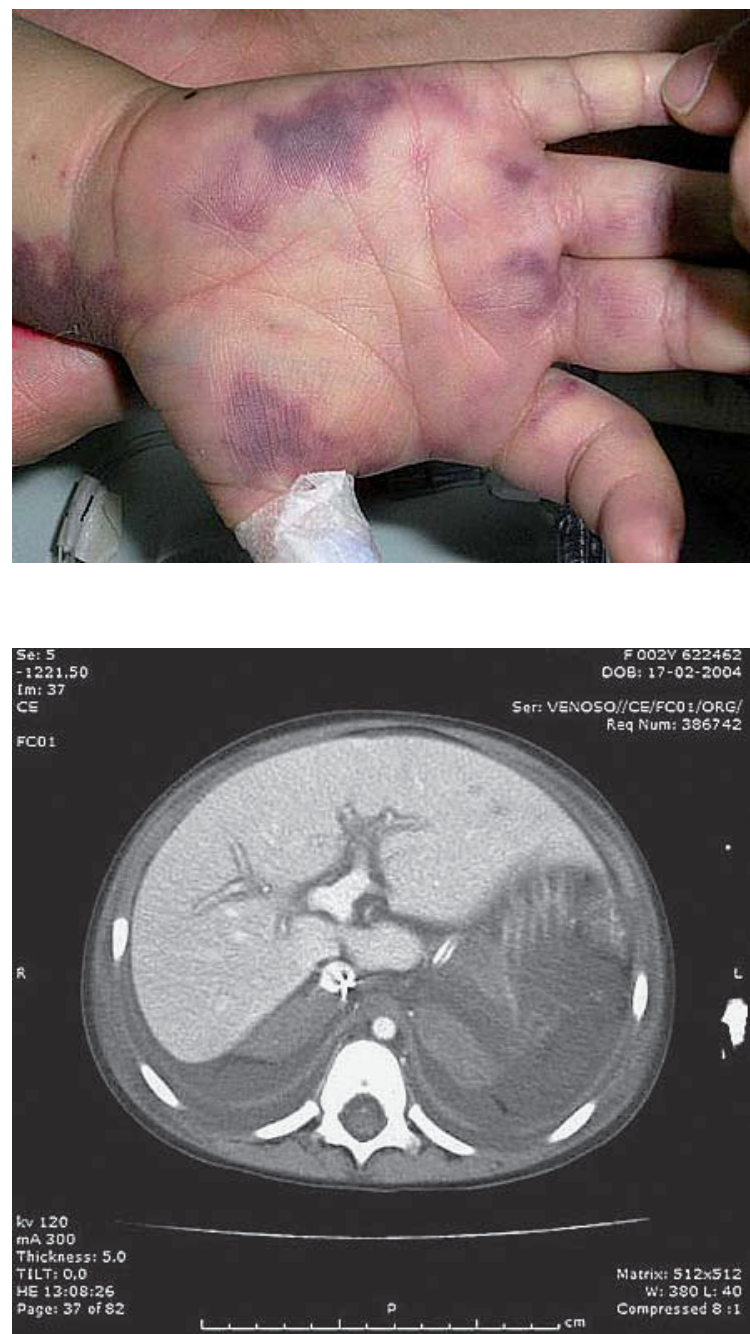

$3.400 / \mathrm{mm}^{3}$ y baciliformes $32 \%$ ), hematocrito $36 \%$, trombopenia de $85.000 / \mathrm{mm}^{3}$, PCR de $57 \mathrm{mg} / \mathrm{l}$ (VN: 0-5 $\mathrm{mg} / \mathrm{l}$ ), protrombinemia de $39 \%$ y TTPA de 119,4" (INR: 2,08). Todo estos hallazgos eran compatibles con shock séptico grave, por lo que se inició ventilación mecánica, monitoreo invasivo y reanimación con volumen (130 $\mathrm{ml} / \mathrm{kg}$ en el primer día), fármacos vasoactivos (norepinefrina, epinefrina, dobutamina) y transfusión de hemoderivados (eritrocitos $60 \mathrm{ml} / \mathrm{kg}$, plasma fresco congelado $30 \mathrm{ml} / \mathrm{kg}$, crioprecipitado $6 \mathrm{U}$, plaquetas $10 \mathrm{U}$ dentro de las primeras 48 horas). Recibió suplementación de corticoesteroides y calcio, alcalinización e infusión continua de insulina. Antibioterapia con vancomicina, (60 mg/kg/día), clindamicina (40 mg/kg/ día) y ceftriaxona (100 mg/ $\mathrm{kg} /$ día). En las primeras 24 horas se evidenció un shock séptico vasopléjico refractario a vasopresores y terlipresina. Evolucionó con síndrome compartimental abdominal, que respondió a descompresión, síndrome de disfunción orgánica múltiple (hepática, renal, SDRA, coagulación intravascular diseminada-CID) que requirió hemofiltración venovenosa continua. No hubo progresión significativa de las lesiones, pero persistió en shock. En consideración a estos antecedentes se realizó en el segundo día de hospitalización ecografía y TAC abdominales, que evidenciaron ausencia de bazo y aumento de volumen suprarrenal bilateral, apoyando el diagnóstico de síndrome de Waterhouse Friderichsen (Figura 2). Se comprobó la presencia de corpúsculos de Howell-Jolly en el frotis sanguíneo. Luego se le administró $2 \mathrm{~g} / \mathrm{kg}$ de inmunoglobulina ev. Se inició ventilación alta frecuencia oscilatoria por SDRA refractario a ventilación mecánica, respondiendo a la posición prona prolongada. Un hemocultivo fue positivo a $S$. pneumoniae, serotipo $19 \mathrm{~F}$, resistente a penicilina (CIM 4,0 $\mu \mathrm{g} / \mathrm{ml})$ y sensible

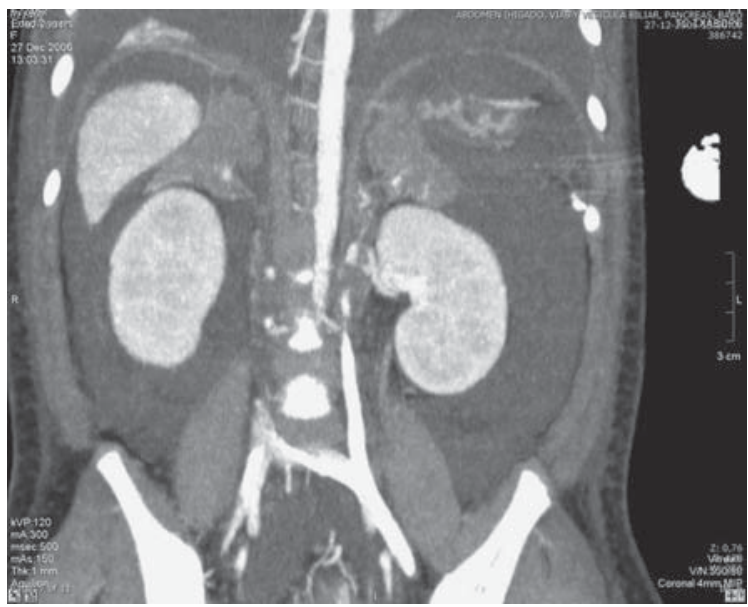

Figura 2. Tomografía axial computarizada de abdomen que revela ausencia de bazo en la fosa esplénica (Figura 2a). Nótese además hemorragia bilateral de suprarrenales (Figura 2b). 
a cefotaxima (CIM 0,75 $\mu \mathrm{g} / \mathrm{ml}$ ). Logró mantener estabilización hemodinámica pero presentó un infarto hemorrágico cerebral bilateral con desarrollo de hemoventrículo e hipertensión endocraneana grave, la cual precipitó su deceso al octavo día de ingresada. La necropsia reveló necro-hemorragia bilateral de glándulas suprarrenales, neumonía bilateral hemorrágica, endocarditis aguda de válvula mitral, émbolos sépticos generalizados; hemorragia intra-parenquimatosa parietal izquierda y hemorragia intra-ventricular en ventrículos laterales. Se confirmó la ausencia de bazo (asplenia congénita).

El estudio inmunológico y de imágenes efectuado a los miembros de la familia arrojó resultados normales.

\section{Discusión}

La asplenia (= ausencia de bazo) puede ser funcional, adquirida (quirúrgica) o congénita. La asplenia congénita se presenta en asociación a otras malformaciones (asplenia congénita sindromática) en más de $90 \%$ de los casos, las que habitualmente determinan el pronóstico del paciente. Dentro de éstas, la más común es la cardíaca compleja. La asplenia congénita aislada (asplenia no sindromática) es menos frecuen$\mathrm{te}^{2}$ pudiendo ser catalogada como familiar o esporádi$\mathrm{ca}^{7}$. Esta última condición fue la presente en nuestro caso.

La asplenia funcional se debe a causas tan variadas como anemia de células falciformes con infartos repetitivos, enfermedades autoinmunes, enfermedad inflamatoria intestinal, patología hematológica crónica y la atrofia por trombosis de la arteria esplénica.

Embriología y función. El desarrollo del bazo se detecta inicialmente alrededor del décimo día de gestación como una serie de condensaciones mesenquimatosas en el mesogastrio adyacente al estómago y a la yema pancreática dorsal, formándose el órgano cuando se fusionan estos agregados. El control embriológico de la esplenogénesis reside en el gen HOX11, localizado en el cromosoma 10. Las células del primordio esplénico se diferencian para formar la cápsula, el tejido conectivo y el parénquima, observándose ya diferenciado durante la quinta semana de gestación. Alrededor de la duodécima semana ya puede reconocerse la forma lobulada del bazo fetal.

El bazo cumple una serie de funciones esenciales en la respuesta inmunológica, tales como fagocitosis, producción de inmunoglobulina M (IgM), complemento, producción y maduración de las células $\mathrm{B}, \mathrm{T}$ y células plasmáticas, como también participa en la destrucción de las tres líneas celulares sanguíneas. Normalmente $\sim 30 \%$ de las plaquetas serán removidas por el bazo; por lo tanto, en condiciones de asplenia es habitual observar leves grados de trombocitosis. Otra función es la selección y extracción de hematíes disfuncionantes y senescentes, identificados a la microscopia por corpúsculos de inclusión como los cuerpos de Heinz (precipitados de globina insoluble), y los cuerpos de Howell-Jolly (fragmentos nucleares). Durante una bacteriemia, además de un alto título de anticuerpos circulantes, es necesaria la presencia del bazo para la producción inmediata de más anticuerpos y para la activación del sistema macrófago-fagocitario ${ }^{8}$. El paciente asplénico se encuentra en clara desventaja respecto a las infecciones invasoras neumocóccicas, especialmente por ciertos serotipos $(12,22,24)$. Así entonces, la pérdida de las funciones previamente señaladas es la base de las alteraciones detectadas en pacientes asplénicos.

Asplenia. Los pacientes asplénicos o hipoesplénicos tienen un riesgo elevado de infecciones graves causadas por bacterias capsuladas ${ }^{8,9}$, como $S$. pneumoniae, Haemophilus influenzae tipo b, y otros microorganismos menos comunes como Streptococcus agalactiae, Staphylococcus aureus, diversas especies de Salmonella, Escherichia coli, Pseudomonas aeruginosa y Capnocytophaga canimorsus; este último, comúnmente asociado a mordeduras de perro, es mucho menos frecuente. Si existe una mayor susceptibilidad a Neisseria meningitidis no está claro aún. Dentro de los parásitos se debe tener presente a Plasmodium falciparum y a los protozoos causantes de babesiosis.

Con frecuencia, estas infecciones, en especial la causada por $S$. pneumoniae, presentan un curso fulminante y cifras de mortalidad que alcanzan hasta el $50 \%{ }^{8}$. Se ha estimado que el riesgo acumulado de padecer una infección durante toda la vida es de $5 \%{ }^{10}$; es mayor en niños bajo 5 años de edad que en los adultos $^{9}$ y en hemoglobinopatías (anemia de células falciformes, talasemia) mayor que en la esplenectomía post-traumática ${ }^{9}$. Es importante tener presente que en esta última situación, el mayor número de episodios de sepsis ocurren generalmente durante los primeros dos años ${ }^{11}$ post cirugía; sin embargo, hasta un tercio de los episodios tienen lugar entre los 5 y 20 años posteriores $^{9}$.

Actualmente existe consenso que los pacientes deben ser evaluados en la búsqueda de una eventual disfunción esplénica en las siguientes situaciones: Infecciones recurrentes o sepsis, especialmente las causadas por microorganismos capsulados, historia familiar de asplenia o polisplenia, evidencia de heterotaxia u otras malformaciones asociadas. 


\section{Tabla 1. Recomendaciones de inmunización en niños asplénicos ${ }^{15,18,19}$}

- La vacunación antineumocóccica en niños asplénicos, dependiendo de la edad, se realizará con la vacuna conjugada heptavalente o utilizando esquemas mixtos con la vacuna conjugada heptavalente y la de 23 polisacáridos

- Si la esplenectomía es programada, se recomienda realizar las vacunaciones al menos dos semanas antes de la misma. Si no hay tiempo antes de la esplenectomía, se recomienda esperar dos semanas después

- La vacunación contra Haemophilus influenzae tipo b está indicada si el niño no está vacunado, siguiendo el esquema de vacunación normal

- La vacunación frente a Neisseria meningitidis se llevará a cabo utilizando la vacuna conjugada tetravalente A-C-Y-W135

- Es recomendable la vacunación anual de la gripe

\section{Métodos diagnósticos}

Laboratorio. El frotis sanguíneo, un examen simple y disponible, puede evidenciar la presencia de cuerpos de Howell-Jolly (fragmentos nucleares), siendo esta la mejor prueba clínica de la alteración funcional del bazo.

Imágenes. En un primer acercamiento, la ecografía abdominal puede ser de gran utilidad para documentar la asplenia. A su vez, la ecografía doppler color nos puede orientar con respecto a la cuantía de flujo sanguíneo e indirectamente al grado de función esplénica. La resonancia magnética y la TAC del abdomen pueden también documentar la ausencia del bazo pero no se consideran de rutina para el diagnóstico, a menos que exista una indicación específica, como en el caso de la heterotaxia.

Estudio funcional. Existen varios métodos para estudiar la función esplénica. Las técnicas radioisotópicas están dentro de las más útiles pero son principalmente empleadas en investigación ${ }^{12,13}$.

\section{Profilaxis antimicrobiana e inmunización}

Antibioterapia. La profilaxis antimicrobiana debe ser iniciada en cuanto se confirme el diagnóstico de asplenia debido al alto riesgo de estos pacientes a padecer infecciones. Para niños bajo dos años de edad se emplea amoxicilina $(20 \mathrm{mg} / \mathrm{kg} /$ día $)$. Habitualmente se recomienda profilaxis hasta los cinco años de edad en pacientes asplénicos o con alteraciones de la función esplénica y al menos un año después de haber sido esplenectomizados quirúrgicamente. La penicilina $\mathrm{V}$ oral disminuye en más de $80 \%$ la incidencia de bacteriemia neumocóccica en niños con anemia de células falciformes ${ }^{10,14,15}$. Sin embargo, en otras formas de asplenia funcional $\mathrm{u}$ anatómica, este hecho no es evidente y algunos autores afirman que la profilaxis puede ser más perjudicial que beneficiosa por la selección de bacterias resistentes ${ }^{10,14,15}$.

Inmunizaciones. En los pacientes con asplenia es- tán indicadas las vacunas frente a $S$. pneumoniae, $H$. influenzae tipo b y $N$. meningitidis (A, C, Y, W135) ${ }^{9}$.

En la Tabla 1 se detallan las recomendaciones de inmunizaciones en niños esplenectomizados o asplénicos.

\section{Síndrome de infección devastadora post esplenectomía-OPSI}

En los pacientes esplenectomizados, se describe un cuadro de sepsis fulminante conocido como OPSI, por su acrónimo en inglés overwhelming post-splenectomy infections. Algunas series nos señalan que el riesgo de presentar OPSI disminuye con el tiempo transcurrido desde la esplenectomía; no obstante, otras series clínicas no muestran una reducción significativa de este $^{8}$. Desde un punto de vista teórico, el riesgo de sepsis puede reducirse en forma significativa con educación, inmunización y profilaxis antimicrobiana. No obstante, en la práctica, estas recomendaciones habitualmente no son seguidas a cabalidad, como tampoco se ha realizado un ensayo clínico para comprobar la eficacia de cualquiera de estas intervenciones.

Un reciente estudio de casos OPSI fue realizado para evaluar factores específicos asociados. Setenta y siete casos fueron reportados, el rango de edad fue entre 3 meses y 87 años. En los esplenectomizados quirúrgicos el intervalo entre la cirugía y el desarrollo de OPSI varió desde 24 días hasta 65 años. Streptococcus pneumoniae causó aproximadamente $90 \%$ de los casos. Sólo 31\% había recibido la vacuna antineumocóccica antes del episodio de OPSI. La mitad de las infecciones neumocóccicas en pacientes inmunizados fueron considerados falla de la vacuna ${ }^{8}$.

El uso de inmunoglobulina endovenosa $(0,4 \mathrm{~g} / \mathrm{kg}$ día por tres días) es discutido. Aunque no se cuenta a la fecha de estudios que demuestren su beneficio, existe una buena base teórica que apoya su empleo ${ }^{16,17}$.

\section{Comentario}

En el presente caso de asplenia congénita que presentó un shock séptico de etiología neumocóccica, complicándose con un síndrome de WaterhouseFriderichsen (SWF) asociado al desarrollo de purpura fulminans, el diagnóstico de la condición de asplenia fue evidentemente muy tardío, pese a ser la paciente portadora de cardiopatía y haber evolucionado por largo tiempo con infecciones recurrentes. Esto impidió tomar las precauciones recomendadas, como también lo tardío de las medidas terapéuticas instauradas, lo cual sin duda influyó finalmente en el pronóstico ominoso. En este caso en particular, la búsqueda sistemá- 
tica de corpúsculos de Howell-Jolly en el frotis sanguíneo y el examen ultrasonográfico del abdomen eran trascendentales en el diagnóstico de asplenia. De haberse demostrado tal condición, el uso de profilaxis antimicrobiana y vacunación antineumocóccica debería haberse establecido.

\section{Resumen}

La condición de asplenia predispone a infecciones invasoras por bacterias capsuladas. Desconocer previamente ese antecedente dificulta y retarda el tratamiento médico. Streptococcus pneumoniae es el agente habitualmente causal de sepsis en estos pacientes. Los individuos asplénicos son particularmente proclives a evolucionar con shock séptico y eventualmente al desarrollo de purpura fulminans, entidad altamente letal. Comunicamos el caso de una paciente con 3 años de edad y antecedente de cardiopatía y neumonías a repetición. Ingresó con compromiso sen- sorial, febril, hipotensa, con púrpura y livedo reticularis. En los exámenes de laboratorio destacaba la presencia de leucopenia $\left(3.400 / \mathrm{mm}^{3}\right)$ trombopenia e hipoprotrombinemia (39\%). Se inició ventilación mecánica, reanimación con volumen, fármacos vasoactivos y antibioterapia con vancomicina, clindamicina y ceftriaxona. Evolucionó con shock séptico refractario y síndrome de disfunción orgánica múltiple. Al segundo día de evolución una ecografía de abdomen comprobó la ausencia de bazo. En el frotis sanguíneo se evidenciaron corpúsculos de Howell-Jolly. Hemocultivo (+) S. pneumoniae resistente a penicilina (serotipo 19F). Un infarto hemorrágico cerebral ocasionó su deceso al octavo día. El estudio necrópsico corroboró la asplenia y evidenció necrohemorragia suprarrenal bilateral. La sepsis en un paciente asplénico puede ser de alguna forma prevenible mediante profilaxis antimicrobiana y vacunación neumocóccica. Dado los antecedentes de la paciente la búsqueda de asplenia era fundamental.

\section{Referencias}

1.- Waldman J, Rosenthal A, Smith A, Shurin S, Nadas A. Sepsis and congenital asplenia. J Pediatr 1977; 50: 555-9.

2.- Halbertsma F, Neeleman C, Weemaes C, Van Deuren M. The absent and vanishing spleen: congenital asplenia and hyposplenism-two case reports. Acta Paediatr 2005; 94: 369-83.

3.- Picard C, Puel A, Bustamante J, Ku C L, Casanova J L. Primary immunodeficiencies associated with pneumococcal disease. Curr Opin Allergy Clin Immunol 2003; 3: 451-9.

4.- Long W A. Ivemark syndrome (cardiosplenic or heterotaxy syndrome). Fetal Neonatal Cardiol 1990: 616-9.

5.- Lynch A M, Kapila R. Overwhelming postsplenectomy infection. Infect Dis Clin North Am 1996; 10: 693-707.

6.- Bertrosian A, Berlet T, Agarwal B. Purpura fulminans in sepsis. Am J Med Sci 2006; 332: 339-45.

7.- Kanthan R, Moyana T, Nyssen J. Asplenia as a cause of sudden unexpected death in childhood. Am J Forensic Med Pathol 1999; 20: 57-9.

8.- Waghorn D J. Overwhelming infection in asplenic patients: current best practice preventive measures are not being followed. J Clin Pathol 2001; 54: 214-8.

9.- Working Party of the British Committee for Standards in Haematology Clinical Haematology Task Force. Guidelines for the prevention and treatment of infection in patients with an absent or dysfunctional spleen. Br Med J 1996; 312: 430-4.

10.- Davidson R N, Wall R A. Prevention and management of infections in patients without a spleen. Clin Microbiol Infect 2001; 7: 657-60.

11.- Holdsworth R J, Irving A D, Cuschieri A. Postsplenectomy sepsis and its mortality rate: actual versus perceived risks. Br J Surg 1991; 78: 1031-8.

12.- Fischer K C, Shapiro S, Treves S. Visualization of the spleen with a boneseeking radionuclide in a child with sicklecell anemia. Radiology 1977; 122: 398.

13. - Heck L I, Brittin G M. Splenic uptake of both technetium-99m diphosphonate and technetium-99m sulphur colloid in sickle cell anemia and thalassemia. Clin Nucl Med 1989; 14: 557-63.

14.- Styrt B. Risk of infection and protective strategies for the asplenic patient. Infect Dis Clin Pract 1996; 5: 94-100.
15.- Pickering L K, Baker J C, Long S S, McMillan J A, eds. American Academy of Pediatrics. Immunization in special clinical circumstances: Children with asplenia. In: Red Book 2006 Report of the Committee on Infectious Diseases. 27th ed. Elk Grove Village, IL: American Academy of Pediatrics 2006: 83-85.

16.- Camel J E, Kim K S, Tchejeyan G H, Mahour G H. Efficacy of passive immunotherapy in experimental postsplenectomy sepsis due to Haemophilus influenzae type b. J Pediatr Surg 1993; 28: 1441-4.

17.- Offenbartl K, Christensen P, Gullstrand P, Prellner K. Prophylactic effect of human immunoglobulin against pneumococcal postsplenectomy sepsis in the rat. Infection 1986; 14: 167-9.

18.- Royal College of Paediatrics and Child Health. Immunisation of the immunocompromised child. Best Practice Statement. February 2002. www.rcpch.ac.uk

19. - National Advisory Committee on Immunization. Immunization in immunocompromised children. Canadian Immunization Guide. Sixth edition. 2002; 20-30. 